\title{
Bio-Inspired Motion Detector Model Simulated on Xilinx ISE
}

\author{
Rekha $\mathrm{G}^{1}$ \\ Arunkumar PChavan ${ }^{2}$ \\ Ravish Aradhya $\mathrm{H} \mathrm{V}^{3}$ \\ ${ }^{1,2,3}$ Department of Electronics and Communication, $R \vee$ college of Engineering, Bangalore 560059, India.
}

\begin{abstract}
In research and scientific applications Bio-inspired motion detection models have been attracted by small-scale unmanned aerial vehicles (UAVs). UAVs are used for surveillance, security, search and rescue mission. Research on autonomous navigation is greatly influenced by the anatomy of flying insect's eyes. The basic Reichardt correlator which seems to be the fundamental part in all insect motion processing is used in finding relative motion. An array of Reichardt correlator is used to correlate between each pixel values of successive frames. Responses obtained from each correlator model are aggregated together which give us the information about the direction of motion. The proposed architecture is synthesized and analyzed for timing and power using Xilinx ISE tool. This architecture can have applications for obstacle detection and motion detection in various fields.
\end{abstract}

\section{Keywords}

Unmanned Aerial Vehicles (UAVs), Bio-inspired, Reichardt correlator, Motion detector.

\section{INTRODUCTION}

An unmanned aerial vehicle (UAV), commonly known as a drone is an aircraft without a human pilot on board. Its flight is controlled either autonomously by computers in the vehicle, or under the remote control of a pilot on the ground or in another vehicle. Historically, UAVs were simple remotely piloted aircraft, but autonomous control is increasingly being employed. They are deployed predominantly for military applications, but also used in a small but growing number of civil applications, such as policing, firefighting and nonmilitary security work, such as surveillance of pipelines. UAVs are often preferred for missions that are too "dull, dirty, or dangerous" for manned aircraft. Surveillance applications include: livestock monitoring, wildfire mapping, pipeline security, home security, road patrol and anti-piracy. The trend for use of UAV technology in commercial aerial surveillance is expanding rapidly with increased development of automated object detection approaches.

Autonomy is commonly defined as the ability to make decisions without human intervention. The goal of autonomy is to teach machines to be "smart" and act more like humans. Early UAVs used during the Vietnam War after launch captured video that was recorded to film or tape on the aircraft. These aircraft often were launched and flew either in a straight line or in preset circles collecting video until they ran out of fuel and landed. After landing, the film was recovered for analysis. Because of the simple nature of these aircraft, they were often called drones. Today's UAVs often combine remote control and computerized automation. More sophisticated versions may have built-in control and/or guidance systems to perform low-level human pilot duties

such as speed and flight-path stabilization, and simple scripted navigation functions such as waypoint following.
Compared to the manufacturing of UAV flight hardware, the market for autonomy technology is fairly immature and undeveloped. Because of this, autonomy has been and may continue to be the bottleneck for future UAV developments, and the overall value and rate of expansion of the future UAV market could be largely driven by advances to be made in the field of autonomy. From this perspective, an attempt is made to develop Bio-inspired motion detection model which can be implemented on FPGA for high speed processing. Bioinspiration is the application of biological methods and systems found in nature to the study and design of engineering systems and modern technology. One of the most eminent motion detection models is the Reichardt correlator model [1] [2] [3] [4] [5]. Flying insects use highly efficient visual strategies to control their self-motion in threedimensional space [6] [7]. Reichardt's findings have contributed to understanding of information processing in insects eye. Optical flow processing in flying insect is very fast and serves as a basis for many motion detection models. Reichardt Correlator model replicates flying insect's visual system for flight control, stabilization and object tracking. In the early 1960's Reichardt deduced an Elementary Motion Detection (EMD) which describes an insects' visual information processing systems. In [8], a low-power VLSI chip which consists of a one-dimensional array of EMDs was proposed to perform motion computation.

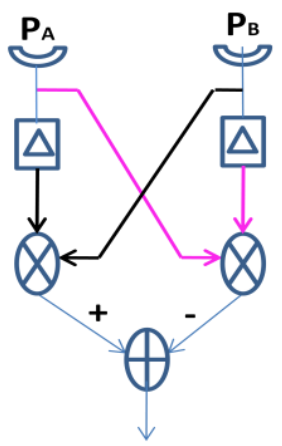

Figure 1: Basic Reichardt detector model A basic Reichardt detector comprises of two mirrorsymmetric subunits as shown in Fig 1. In each sub-unit one of the signals received from photoreceptor is delayed using a low-pass filter or a delay line. The delayed signal is then correlated with the signal received from the neighboring photoreceptor. The response is the difference between the two correlated signals.

The model yields a positive response if an object passes from left to right. This is because for left to right movement the left subunit is actuated first then the second subunit is activated with a delay. This makes the left multiplier to produce a nonzero value and the right multiplier produces zero value. 
Finally the difference between these values gives a positive value indicating left to right movement. Similarly rives a negative response for opposite direction.

\section{PROPOSED WORK}

The proposed architecture is as shown in figure 2. The architecture consists of ROM, Counter, RAM, Multiplexer, Finite State Machine, Serial in Parallel out and an array of Reichardt motion detection model.

Each ROM stores the image pixel values which are fed as input to be processed. Counters are used to provide address location for memories. In this case output from ROM can be directly fed as input to SIPO block. RAM block can be used when input is to be received externally. Multiplexer is used to select the frame. First frame pixel values are given to SIPO block which are then sent parallel to the motion detection model. Now each Elementary Motion Detection model has an input. After a small delay multiplexer is switched to the second frame which then appear as input to each EMD cell. At this point the previous frame values are also delayed by an amount of time required to receive Each Reichardt model used in the array consists of three EMD cells to provide horizontal, vertical and diagonal responses as shown in Fig 3.

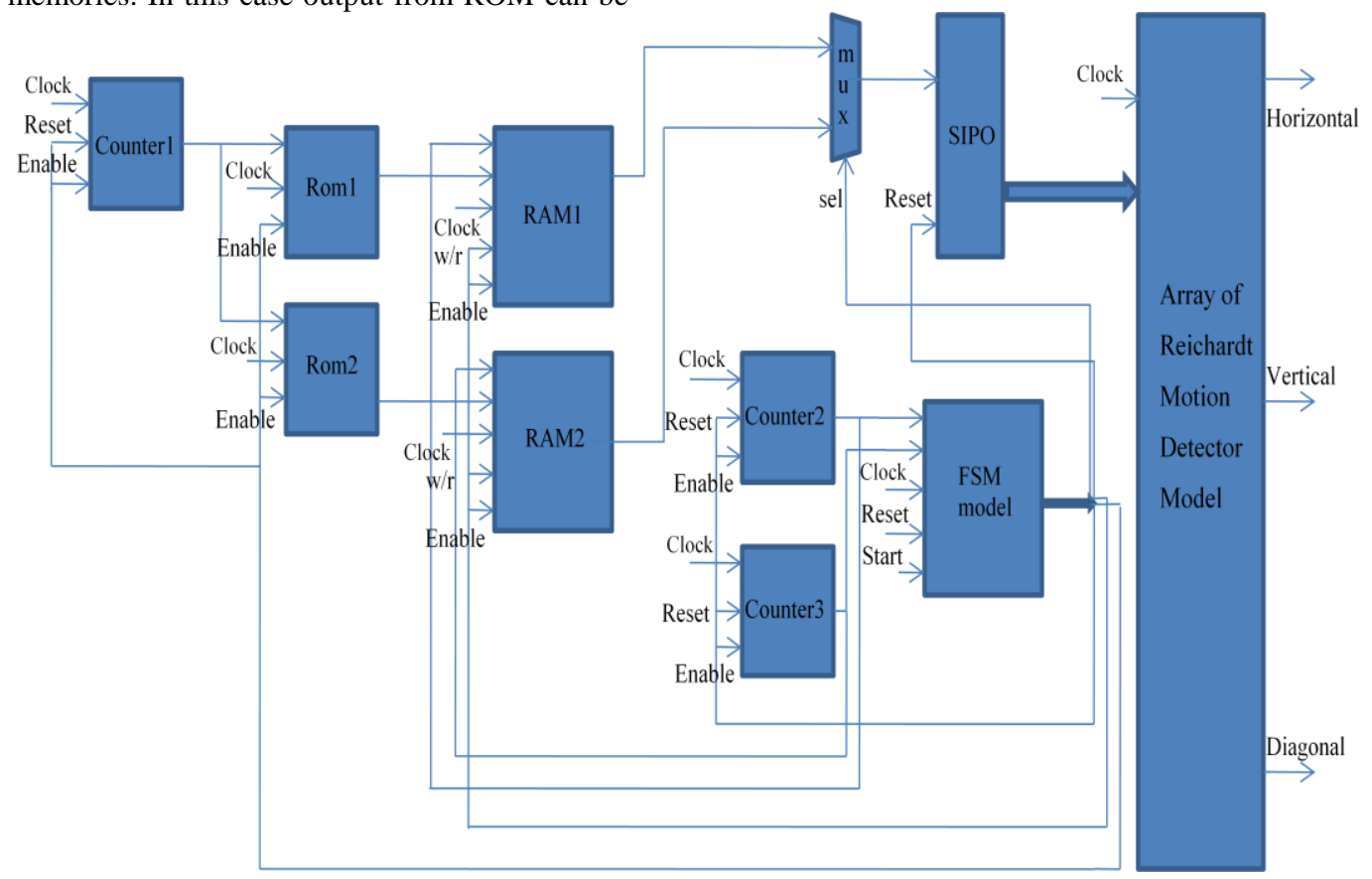

Figure 2: Proposed Architecture to process the pixel values for Motion detection.

Pink color indicates signals with no delay and black color indicates delayed signals. The architecture uses SIPO block to reduce the number of input wires. This makes it simpler to implement the architecture on FPGA platform.

\section{SIMULATION RESULTS}

Verilog code is written for the proposed architecture and is simulated using Xilinx ISE tool. Figure 4 shows the schematic view of the Reichardt correlator model. Figure 5 shows the simulation result obtained for motion detection. 1 and -1 response for the signal $\mathrm{H}$ indicates horizontal left to right and right to left movement. -1 and 1 values of signal $\mathrm{V}$ indicates vertical top to bottom and bottom to top movement. Similarly -1 and 1 for signal D shows diagonal down movement and diagonal up movement. Simulation result for six directions is shown.

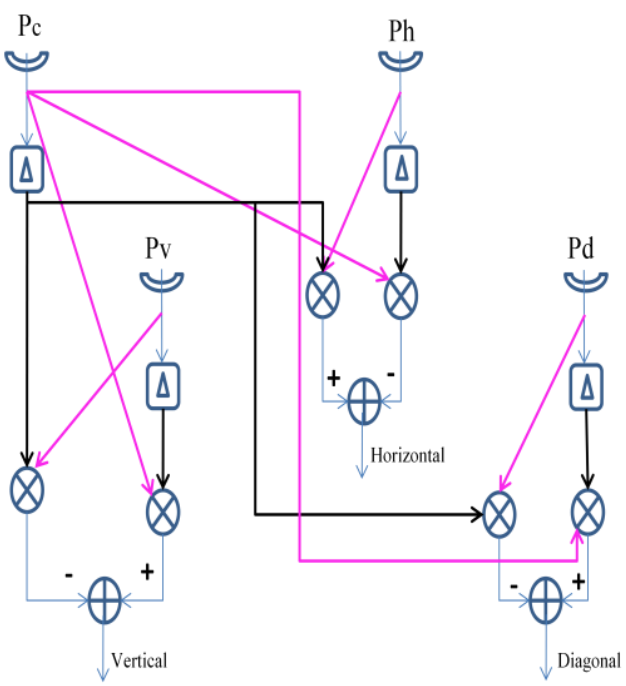

Figure 3: Reichardt model for horizontal, vertical and diagonal movement detection. 


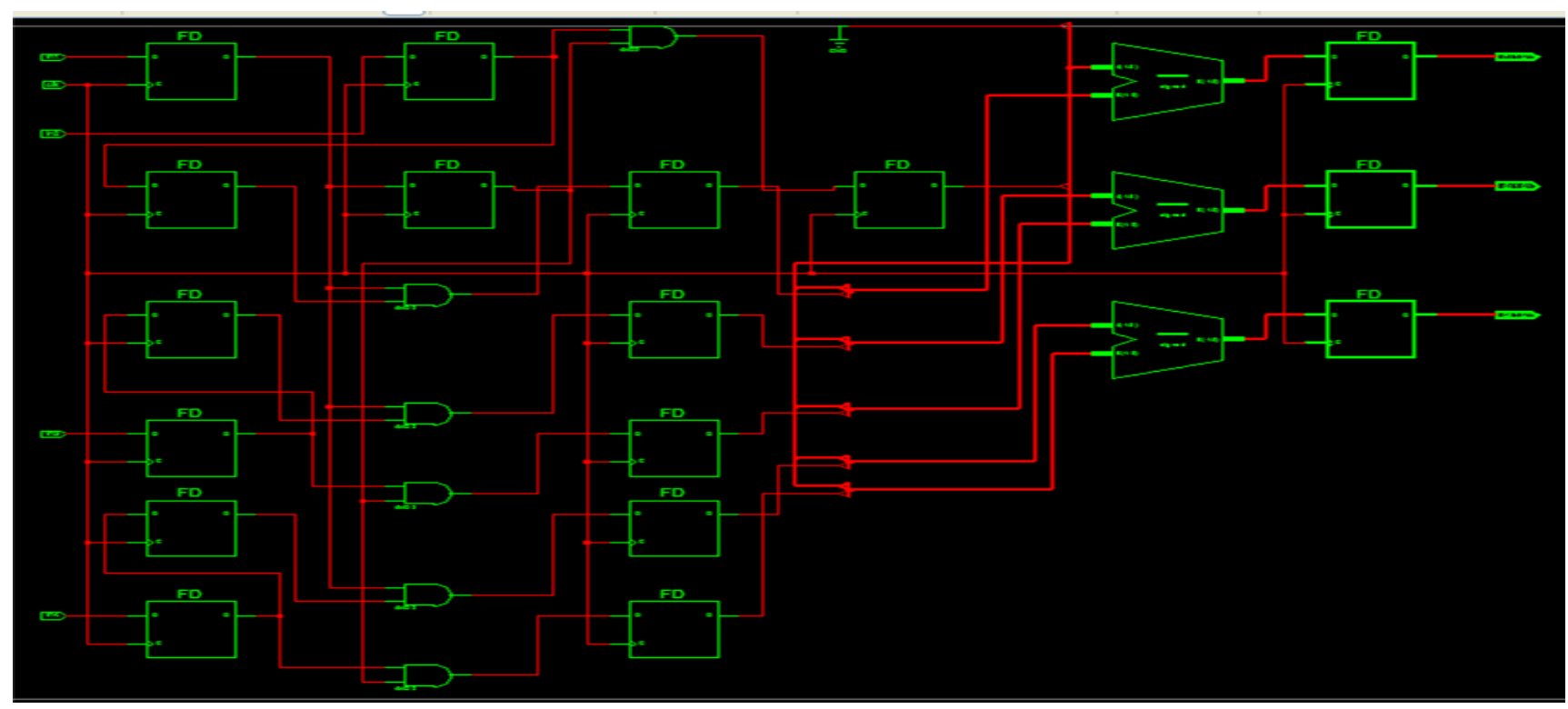

Figure 4: Schematic view of Reichardt correlator model.

\begin{tabular}{|c|c|c|c|}
\hline \multicolumn{4}{|c|}{$\begin{array}{l}\text { Fur Simulation } \\
\text { File Edit View }\end{array}$} \\
\hline \multicolumn{4}{|c|}{$\begin{array}{l}\text { Current Simulatior } \\
\text { Time: } 1000 \text { ns }\end{array}$} \\
\hline \multicolumn{4}{|c|}{$+61 H[4: 0]$} \\
\hline \multicolumn{4}{|c|}{$+6 / v[4: 0]$} \\
\hline \multicolumn{4}{|c|}{$+510[4: 0]$} \\
\hline \multicolumn{4}{|c|}{ b/ } \\
\hline \multicolumn{4}{|c|}{ I } \\
\hline \multicolumn{4}{|c|}{ b } \\
\hline \multicolumn{4}{|c|}{ क人 } \\
\hline \multicolumn{4}{|c|}{ क्र $\mathrm{c}$} \\
\hline \multicolumn{4}{|c|}{ or } \\
\hline \multicolumn{4}{|c|}{ क्रा d1 } \\
\hline \multicolumn{4}{|c|}{ का 12} \\
\hline \multicolumn{4}{|c|}{ ord } \\
\hline & $5 \sqrt{1} d 4$ & & \\
\hline
\end{tabular}

ench Simulation window

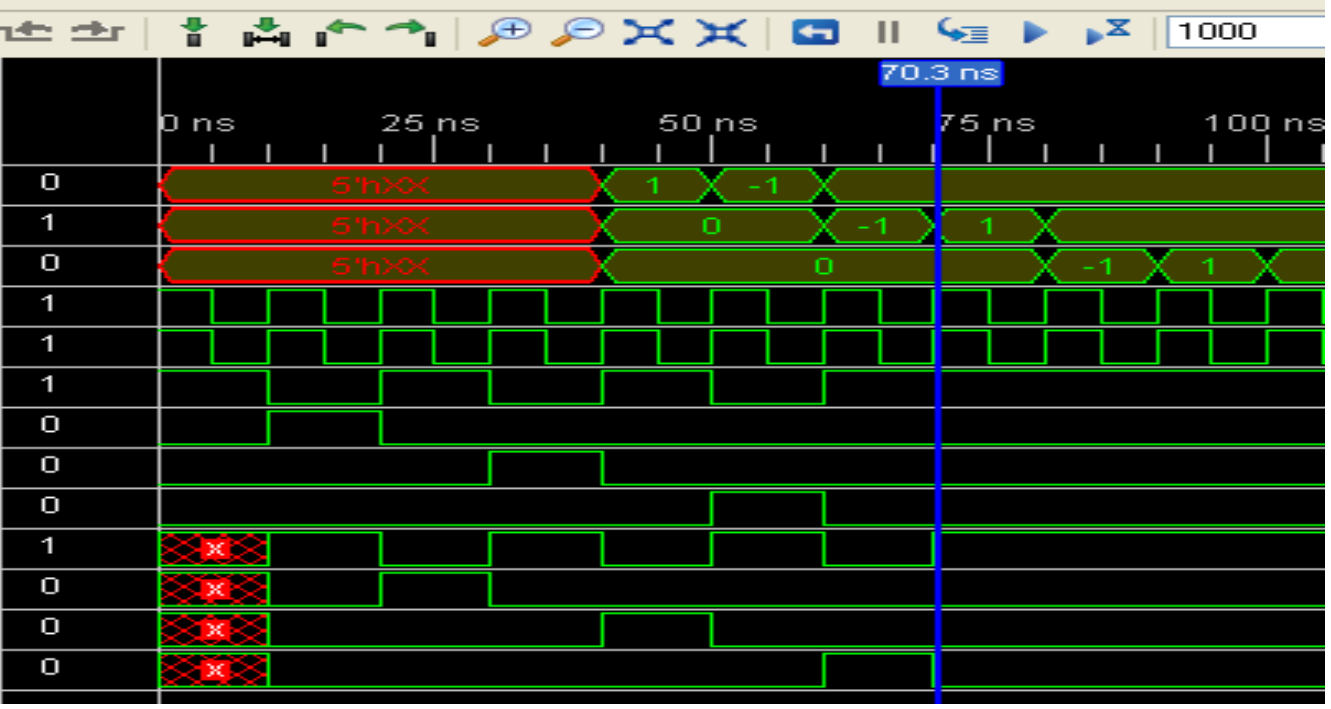

Figure 5: Simulation result for motion detection.

After receiving the input three clock cycles are required to compute the motion direction. Horizontal component of each EMD model are added row wise and then aggregated together. The entire vertical components are added column wise and aggregated as a single output. In a similar way all the diagonal components are added diagonally and aggregated as a single output.

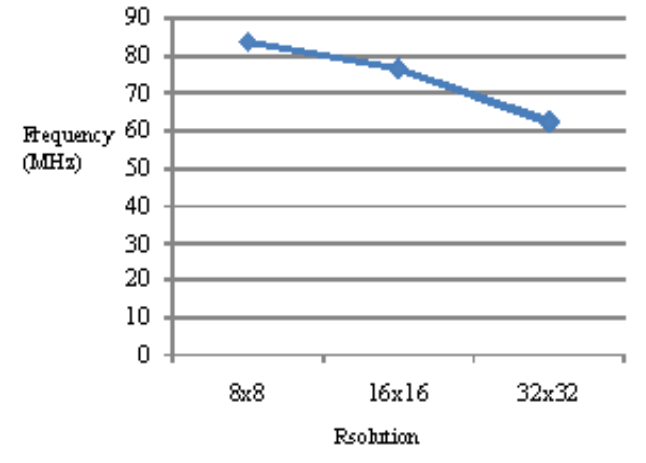

Figure 6: Variation of Frequency with the Resolution
As the image resolution increases the maximum frequency of operation decreases. This variation is shown in the figure 6 . Frequency shown is in $\mathrm{MHz}$ range. Minimum input arrival time before clock is $0.980 \mathrm{~ns}$.Maximum output required time after clock: 2.826ns.

\section{CONCLUSION}

In this paper, the biologically inspired Reichardt correlator model is used. The proposed architecture uses an array of Elementary motion detection model for detecting moving object direction. The architecture uses SIPO block. This reduces the number of IOBs for implementing the architecture on FPGA platform. If the frame is divided into $8 \times 8$ blocks for processing, then it can work with a maximum frequency of 83.512MHz. Minimum input arrival time before clock is $0.980 \mathrm{~ns}$.Maximum output required time after clock is $2.826 \mathrm{~ns}$.

\section{REFERENCES}

[1] M. Egelhaaf and A. Borst, "A look into the cockpit of the fly: Visual orientation, algorithms, and identified neurons," The Journal of Neuroscience, vol. 13, pp. 4563-4574, 1993. 
[2] W. Reichardt and M. Egelhaaf, "Properties of individual movement detectors as derived from behavioral experiments on the visual system of the fly," Biological Cybernetics, vol. 58, pp. 287-294, 1988.

[3] A. Borst and M. Egelhaaf, "Principles of visual motion detection," Trends Neurosci, vol. 12, pp. 297-306, 1989.

[4] T. Neumann and H. Bulthoff, "Insect inspired visual control of translator flight," Advances in Artificial Life. 6th European Conference, ECAL 2001. Proceedings, vol. 2159, pp. 627-636, 2001.

[5] C. M. Higgins and S. A. Shams, "A biologically inspired modular VLSI system for visual measurement of selfmotion," IEEE SENSORS JOURNAL, vol. 2, pp. 508$528,2002$.

[6] A. Borst and J. Haag, "Neural networks in the cockpit of the fly," Journal of Comparative Physiology A, vol. 188, pp. 419-437, 2002.

[7] J. Haag and A. Borst, "Neural mechanism underlying complex receptive field properties of motion-sensitive interneurons," Nature Neurosci, vol. 7, pp. 628-634, 2004.

[8] S.-C. Liu, "A neuromorphic a VLSI model of global motion processing in the fly," IEEE TRANSACTIONS ON CIRCUITS AND SYSTEMS, vol. 47, pp. 1458-1467, 2000.

\section{AUTHORS PROFILE}

A. Rekha G born on March, 15th, 1990 in Karnataka, India, obtained her B.E degree in Electronics and Communication Engineering from Visvesvaraya Technological University (VTU), Belgaum, India. Currently pursuing M.Tech in VLSI Design and Embedded Systems. Her areas of interest are VLSI design, Image Processing, and Digital electronics.
B. Arunkumar. P Chavan born on July, 4th, 1987 in Karnataka, India, obtained his B.E degree in Electronics and Communication Engineering from Visvesvaraya Technological University (VTU), Belgaum, India. Currently pursuing M.Tech in VLSI Design and Embedded Systems. His areas of interest are VLSI design, Analog circuit design and digital electronics.

C. Ravish Aradhya H. V., currently working as Associate professor in Electronics \& Communication Engineering Department at RV College of Engineering, Bangalore 560 059. The author is associated with this premier institution for the past 18 years. With a vast 22 years of teaching experience, the author has understood the basic needs of student community in learning the technology. He has set up various laboratories like Digital circuits lab, Microprocessors lab, Micro controllers lab, Computer communication Networks lab, Analog Circuits lab and prepared study material for the same in the department. He received " BEST TEACHER" award for Excellence in Teaching from CARRIER LAUNCHER (INDIA), Ltd. He has guided several projects that are executed by his students for various reputed organizations like ISRO, INTEL, TI, ADA, ADE, BEL, NAL, ETC. The author headed the team of Computer Organization subject in Visweswaraiah Technological University's EDUSAT program.

His research area is low power VLSI design and he has submitted his research thesis on Reversible logic design to Visvesvaraya Technological University (VTU), Belgaum, Karnataka for evaluation. The author, to his credit has 11 International and 17 National papers presented. His areas of interest include the Electronic Devices and circuit theory, VLSI design, Microprocessor and Microcontroller applications, embedded systems and Computer networking 